\title{
Substrate-interface interactions between carbon nanotubes and the supporting substrate
}

\author{
R. Czerw, ${ }^{1}$ B. Foley, ${ }^{1,2}$ D. Tekleab, ${ }^{1}$ A. Rubio, ${ }^{3}$ P. M. Ajayan, ${ }^{4}$ and D. L. Carroll ${ }^{1}$ \\ ${ }^{1}$ School of Materials Science and Engineering, Clemson University, Clemson, South Carolina 29634 \\ ${ }^{2}$ Department of Physics, Trinity College Dublin, Dublin 2, Ireland \\ ${ }^{3}$ Departamento de Fisica de Materiales, UPV/EHU, Donostia International Physics Center (DIPC), Centro Mixto CSIC-UPV/EHU, \\ 20018 San Sebastian, Spain \\ ${ }^{4}$ Department of Materials Science and Engineering, Rensselaer Polytechnic Institute, Troy, New York 12180-3590
}

(Received 30 October 2001; revised manuscript received 3 June 2002; published 30 July 2002)

By utilizing the current transients in scanning tunneling spectroscopy, the local interfacial electronics between multiwalled carbon nanotubes and several supporting substrates has been investigated. Voltage offsets in the tunneling spectra are directly correlated with the formation of a dipole layer at the nanotube-substrate interface, strongly suggesting the formation of interface states. Further, a systematic variation in this local potential, as a function of tube diameter, is observed for both metallic substrates $(\mathrm{Au})$ and semimetallic substrates (graphite). In both cases, for tubes with diameters between $\sim 5 \mathrm{~nm}$ and $30 \mathrm{~nm}$, the interfacial potential is nearly constant as a function of tube diameter. However, for tube diameters $<5 \mathrm{~nm}$, a dramatic change in the local potential is observed. Using ab initio techniques, this diameter-dependent electronic interaction is shown to derive from changes in the tube-substrate hybridization that results from the curvature of the nanotubes.

DOI: 10.1103/PhysRevB.66.033408

PACS number(s): 61.46.+w, 81.07.De, 68.37.Ef

Over the last several years, an astonishing number of experimental determinations of the electronic transport properties of single-walled carbon nanotubes (SWNT's) and multiwalled carbon nanotubes (MWNT's) have been made ${ }^{1-3}$ and correlated with a number of theoretical predictions. ${ }^{4}$ With only a few exceptions, the experimental focus has been on nanotubes supported on a substrate of some type, and in all cases a system of contacts has been employed using traditional interconnect materials such as $\mathrm{Cu}, \mathrm{Au}, \mathrm{Pt}$, and $\mathrm{Ag}$. Thus, all the experimental information we currently have on the electronics of carbon nanotubes involves experimental designs that use metal/semiconductor-nanotube interfaces, and in most cases these interfaces include the length of the tube through the support. Clearly an important part of any interpretation of transport results must include interactions that may exist between the nanotube and the support substrate and contacts. ${ }^{5}$ Recent theoretical studies have addressed Fermi-level alignment in Au-SWNT systems and have suggested that a charge transfer should exist at the

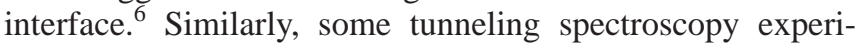
ments have hinted at the existence of charge transfer between the gold and nanotube systems. ${ }^{7}$ However, despite the importance of local interface interactions in transport measurements, no direct determination of its variation with tube diameter, tube chirality, etc., is yet available. In this paper we present an investigation of the interfacial electronic structure between MWNT's and a number of support substrates using current transients in scanning tunneling spectroscopy (STS). These studies strongly suggest that charge transfer at the metal/MWNT interface results from the formation of interface states in analogy to bulk Schottky barriers. Further, the variation of local interface potentials with tube diameter is nearly constant as expected for large-diameter tubes. However, a surprisingly large variation in interface potentials occurs at tube diameters smaller than approximately $5 \mathrm{~nm}$. These variations may result from differences in local hybrid- ization at the metal-nanotube interface caused by the different curvatures of the nanotube walls.

Measurements of the contact potential of three support substrates will be described and compared here: $\mathrm{NiO}, \mathrm{Au}$, and HOPG (highly oriented pyrolytic graphite). In the first case, the $\mathrm{NiO}$ substrates are thin oxide films $(5.0 \mathrm{~nm})$ thermally grown on single-crystal (111) $\mathrm{Ni}$. In the second case, $\mathrm{Au}$ was sputter deposited on annealed mica and then "flamed," leaving a terraced (111) surface for contact with the tubes. Scanning tunneling microscope (STM) imaging of these substrates yielded atomic resolution. After substrate preparation, arc-grown MWNT's, ${ }^{8}$ ultrasonically dispersed in ethanol for $5 \mathrm{~min}$, were deposited. The sample was transferred into UHV (ultrahigh vacuum, $<10^{-10}$ Torr) and outgassed at $300^{\circ} \mathrm{C}$ while adsorbate desorption was monitored to ensure that the substrate was clean. Tunneling conditions used for STM imaging were $20 \mathrm{pA}$ and between 200 and 500 $\mathrm{mV}$. In the case of HOPG substrates, HOPG was cleaved in air and an ultrasonicated solution of nanotubes in ethanol was deposited as above. STM imaging at $20 \mathrm{pA}$ and $300 \mathrm{mV}$ allowed atomic resolution of the substrate and of the supported tubes. Transmission electron microscopy images were correlated to STM micrographs to ensure that the same distribution of diameters of the MWNT's was observed (approximately 2-30 nm). Z-scale calibration of the STM was carried out using known step heights of the Au (111) surface. Finally, work function determinations were compared to the reported values over the clean substrates as a check of $Z$ calibration.

To investigate the nanotube-substrate interface, tunneling spectra $(I-V)$ were acquired both on the clean support substrate and at points on the tube, simultaneously with imaging. In our case, the spectra are acquired by turning off the feedback over the point of interest, ramping the voltage very rapidly, and collecting the current. The rapid ramp rate of the voltage results in current transients that offset the tunneling 

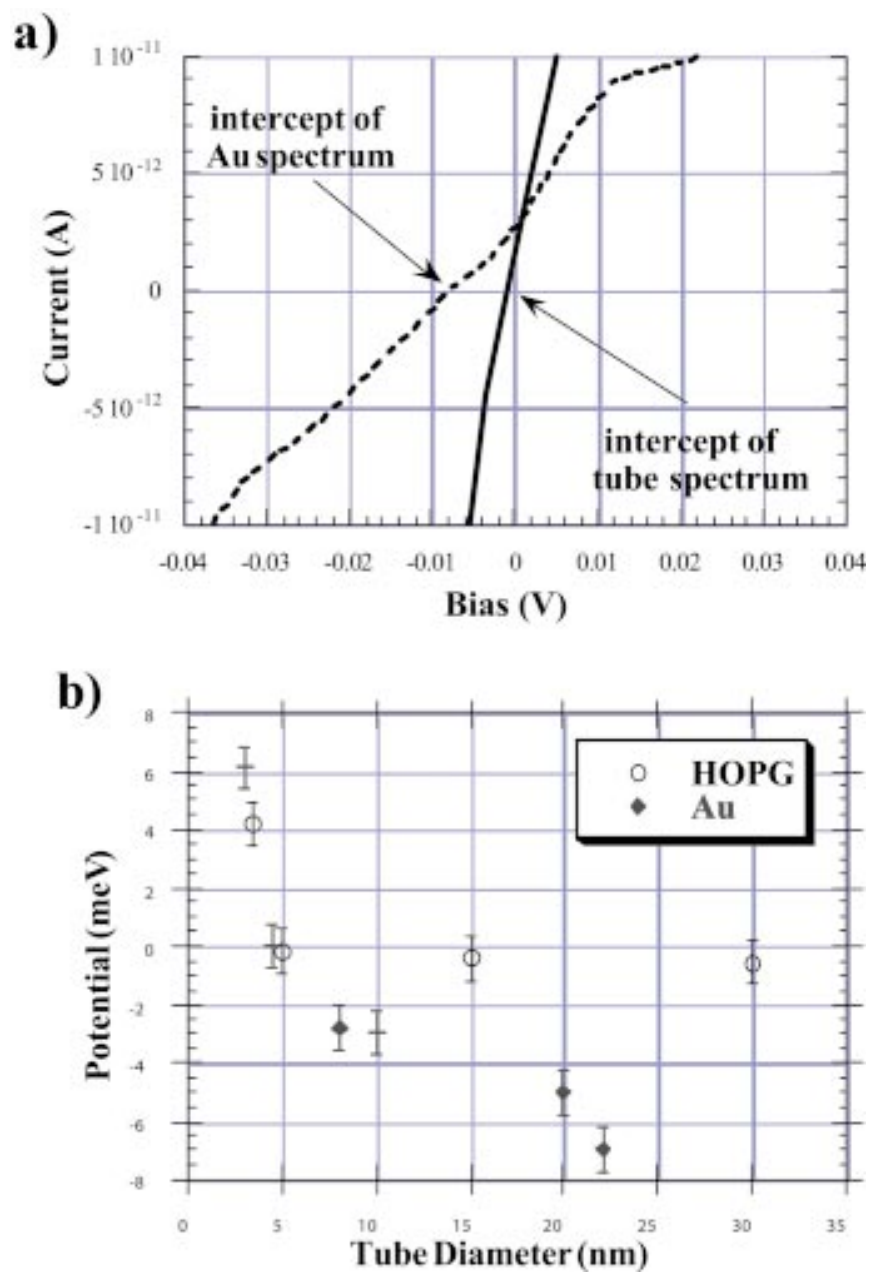

FIG. 1. (Color) (a) Tunneling spectra were collected over the substrate and over the tube for each measurement made. The offsets in tunneling spectra are due to the capacitance of the junction plus local potentials within the junction. Subtracting the shift observed for the $I-V$ curve on the tube from that observed over from the substrate should give zero unless there are extra potentials within the system. (b) The contact potential as determined from the offset in the "high ramp rate" tunneling spectra is strongly dependent on the diameter of the nanotube as shown here. The Au-nanotube samples and the HOPG-nanotube samples are shown.

spectra as shown in Fig. 1(a). The $R C$ time constants associated with capacitances, contact potentials, filter, etc., in the tunneling microscope are very short compared to those of the tunneling junction. ${ }^{9}$ Therefore, the transient-induced shift in the $I-V$ spectra can be associated only with capacitances and potentials within the tunneling junction. The current-axis intercept point $(V=0)$ is well known to be related to the capacitance of the junction from the equation $q=V C$, giving $d q / d t=C d V / d t+V d C / d t=C d V / d t$. The point at which the spectrum crosses the $V$ axis $(I=0)$ is related to the local potentials across the junction and corresponds to the bias offset for which the microscope must compensate to prevent the "discharge" of the junction. This includes the voltage drop across the tip-sample capacitor, as well as any local potentials such as the contact potentials. ${ }^{10}$ The spectra and intercepts for a 22.0-nm-diam tube supported on $\mathrm{Au}$ are shown in Fig. 1(a).

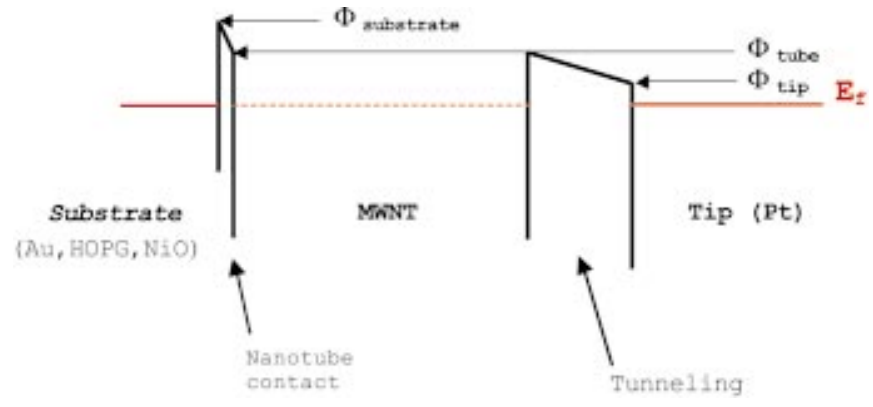

FIG. 2. (Color) An energy diagram of the substrate-nanotube-tip system. In the ideal case where no interface state exists, there should be a simple alignment of Fermi levels and the tube contributions to the potentials within the junction should cancel.

Naturally, we would expect that the comparatively large potentials associated with the capacitive junction and the work function mismatch of the substrate and the Pt tip would dominate the $I-V$ shift. That is, when a nanotube is placed in the tunneling junction it would have little effect outside of changing the junction capacitance through geometry (which would be negligible). This is illustrated in the energy diagram of Fig. 2. Clearly, the contact potentials within the junction-nanotube system will cancel the nanotube component as $\left(\phi_{\text {substrate }}-\phi_{\text {tube }}\right)+\left(\phi_{\text {tube }}-\phi_{\text {tip }}\right)=\phi_{\text {substrate }}-\phi_{\text {tip }}$. However, notice that the intercepts in Fig. 1(a) for the clean $\mathrm{Au}$ surface and the nanotube-Au system are significantly different. ${ }^{11}$ In fact, the same is true for the NiO-MWNT system using 20-nm-diam nanotubes. Specifically, in the Aunanotube system, with a 10 -ms voltage step time (the step width during the voltage ramp) and a 3-ms sample delay time (see Ref. 11), a 20-nm nanotube will exhibit a difference in $V$-intercept shift of 0.075 from that measured on the clean substrate (just subtracting the two intercepts). In the $\mathrm{NiO}$ system, using the same ramping conditions, the shift difference is $0.170 \mathrm{~V}$. For HOPG substrates, large diameter nanotubes give the same value of the voltage offset as the clean substrate. This suggests that there exists local trapped charge at the Au- and NiO-nanotube interfaces while at the HOPG-nanotube interface there are no extra potentials. Using an analogy to bulk contacts, these interfaces then behave as though there are interface states formed between the nanotubes and the substrates [the rapidly ramped $I-V$ is somewhat analogous to capacitance voltage $(\mathrm{CV})$ curves]. ${ }^{12}$ No such interface states appear in the HOPG-nanotube system for large-diameter tubes as would be expected since this looks very much like graphite on graphite.

Twenty tubes supported by Au were studied in this way with diameters that ranged from approximately $3 \mathrm{~nm}$ to 30 $\mathrm{nm}$. The step width used in the voltage ramp was $100 \mathrm{~ms}$ and the delay time was $5 \mathrm{~ms}$. The difference in voltage shifts from the support substrate was determined for these tubes by the method above (subtracting the voltage intercept with the tip over the clean substrate from the intercept with the tip over the tube) and is shown in Fig. 1(b) as a function of tube diameter. Notice that from approximately $10 \mathrm{~nm}$ to $30 \mathrm{~nm}$ diameter, this difference is practically constant. Between 5 and $10 \mathrm{~nm}$ diameters, there is a strong variation in the difference in voltage intercepts. Below $5 \mathrm{~nm}$ the differences in 
the intercepts actually reverse sign. Again, we note that this measurement gives the relative variation in the local potential at the nanotube-substrate interface. Clearly, as the nanotube diameter changes, the local potential varies sharply and actually reverses sign at $5 \mathrm{~nm}$. Figure 1(b) also shows similar measurements performed on HOPG substrates. While the overall difference in the voltage intercepts is $0 \mathrm{~V}$ for large nanotubes as expected, a sharp variation in the local potential is observed at around $5 \mathrm{~nm}$ also. On HOPG, fewer tubes were studied (six), and we note that the values for the larger tubes are not exactly $0 \mathrm{eV}$ as would be expected (graphite on graphite) due to the slight difference in the capacitance between the tip-substrate and the tip-nanotube-substrate junctions.

To understand this behavior, there are two geometrical factors that must be estimated. The first is the variation in the contact area of the tube to the substrate. This changes with tube diameter and will alter the total charge at the interface in direct proportion to the diameter. A simple estimate of the change in contact area requires a guess as to how close the tube wall must be to be considered in contact. From simple geometrical arguments we get area of contact/(unit length) $=(2 R)\left\{\cos ^{-1}[(R-h) / R]\right\}$, where $R$ is the tube radius and $h$ is the height of any point on the tube above the substrate. Here $h$ must be of the order of the $p_{z}$ orbitals $(0.1 \mathrm{~nm})$, and thus the change in contact area is minimal for the MWNT's studied. The second geometrical factor to consider is the effect of the tube diameter on junction capacitance. The capacitance change for the tip-tube-substrate geometry was calculated using classical electrostatics. The total variation in junction capacitance was smoothly varying for tubes of the diameter used in our measurements and also quite small. Thus, we assume in this measurement that this effect is nearly constant. While these geometrical factors could clearly account for the smoothly varying potential associated with large tube diameters, they fail to explain the drastic changes observed below $5 \mathrm{~nm}$. Neither can they account for the observed sign changes in the potential. This suggests that the local charge at the nanotube-substrate interface varies strongly with tube diameter. Further, it is likely that these variations result from changes in the interface electronic structure such as hybridization shifts, shifts in local orbital occupancy, or inner tube spacing changes for the smaller-diameter MWNT's (Ref. 13) that may result in shifts in the overall charge density on the outer shell, and not from pure geometric factors.

The results in Fig. 1(b) point to the existence of trapped charge at the nanotube-substrate interface. This charge is beyond what would normally be found in the Schottky compensation for work function mismatch and must be related to a state that exists at that interface but not found at the tunneling junction (nanotube-Pt). In the regime of large tube diameters, it seems that such states form in the case of NiOand Au-nanotube contacts, but not in the case of HOPGnanotube interfaces. However, to address the transition from large-diameter limit to small-diameter limit, we have performed first-principles pseudopotential density-functional calculations of a perfect SWNT $(5,5)$ supported on a $\mathrm{Au}$ (111) surface and on a graphite surface and compared this to the electronic structure of a single graphene sheet on top of

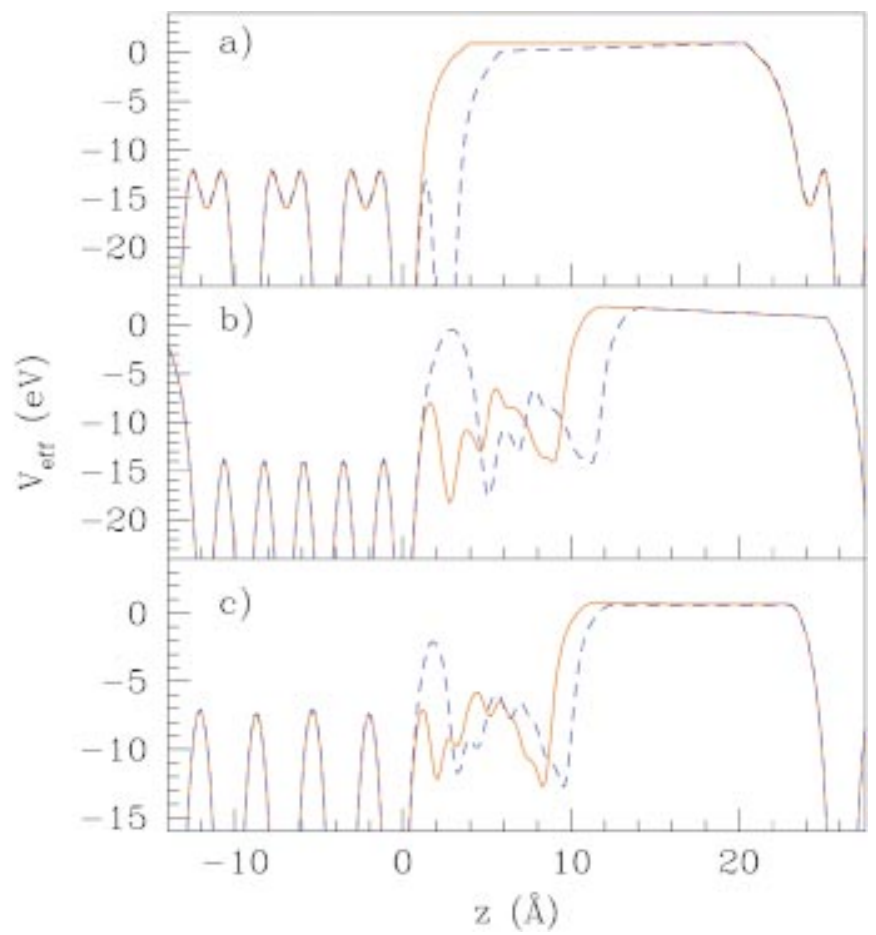

FIG. 3. (Color) Ab initio calculations of the effective electron potential in a supercell geometry for a graphene layer and a $\mathrm{C}(5,5)$ tube on $\mathrm{Au}(111)$ (a) and (b), respectively, and $\mathrm{C}(5,5)$ on graphite (c). In all cases we plot the effective potential along a line perpendicular to the gold surface. Due to computation limitations, we have only considered six gold layers in (b). The results in (a) correspond to the isolated $\mathrm{Au}(111)$ surface (solid line) and a single graphene sheet supported on it (dashed line). The formation of a dipole barrier due to the difference in work functions of graphite and gold is clear. When a small diameter single-wall nanotube is supported on $\mathrm{Au}(111)$ (b) there is a clear change of sign of the barrier. We have plotted the effective potential for two distances, $0.22 \mathrm{~nm}$ (solid line) and $0.34 \mathrm{~nm}$ (dashed line), of the tube with respect to $\mathrm{Au}(111)$. This is directly connected with the observed change of sign in the interface potential in Fig. 1(b). In (c) we plot the same as in (b) but this time for a tube supported on graphite. Notice, here, that there is no dipolar barrier formed.

both substrates (our model for the large-diameter limit). For the case of a graphene sheet on gold, we clearly observe the formation of a simple Schottky barrier dipole Fig. 3(a). This result is independent of the relative orientation of the graphene sheet with respect to the gold substrate. The computed graphene work function of $4.42 \mathrm{eV}$ is very close to the HOPG values and it is smaller than the computed work function of $\mathrm{Au}, 5.3 \mathrm{eV}$. We have also checked that as we increase the number of graphene layers, the work function increases with a small (few meV) dependence on stacking ( $A B$ versus $A A$ or random) sequence. (A small, negative, contact potential for large-diameter MWNT's on HOPG will result from this difference in work functions due to the random nature of the tube-layer stacking. ${ }^{14}$ ) This is compared to the formation of an interface potential at the small-diameter nanotube- $\mathrm{Au}$ contact shown in Fig. 3(b). Because the contact "distance" is not well determined, the graphs compare the corresponding one-electron effective potential for two distances of the tube 
with respect to the substrate $(0.22$ and $0.34 \mathrm{~nm})$. In both cases we see that the small-diameter nanotube on Au develops the opposite barrier height in the vacuum region as that of the graphene sheet on Au. Between these two extremes, as the nanotube radii are increased, there should be a transition from one polarity to the other. This distinct behavior could explain the observed change of sign in the local interface potential. Physically, there is a charge distribution at the interface that leads to either covalentlike or ioniclike bonding states. The redistribution of charge density is still observable in the effective potential even for a tube far from the $\mathrm{Au}$ (111) surface [dashed line in Fig. 3(b)]. This electronic charge distribution and effective electronic screening are controlled by the overlap between gold $d$ states and the outer (interlayer and surface) tube states. These results assume perfect tubes and that the outer layer is the only important one as suggested by other studies on electronic transport in MWNT's. ${ }^{15}$ Contributing to this interface charge redistribution is the opening of small pseudogaps at the Fermi level as a consequence of breaking the tube-mirror symmetry. Recently, the charge density redistribution has been shown theoretically ${ }^{7}$ to align the Fermi level at the metal-carbon nanotube interface and its influences on STM spectroscopy have been explored. We note that these authors work in the limit of large tube diameter where the difference in work functions is the key component determining the contact potential. Further, as argued above, to first order the overall effect of the nanotube in the tunneling junction cancels (aside from capacitive effects) unless state-induced Fermilevel pinning occurs at the interface.

In the case of the HOPG substrate, the bonding is weaker as seen from the effective potential plotted in Fig. 3(c) for small-diameter tubes. No Schottky-like dipole is observed for the particular set of relative orientations chosen. However, the vacuum plateau is a little above the zero of energy that we have taken as the graphitic vacuum level. This indicates the possible change of sign in the contact potential with respect to the case where a single graphene sheet is deposited on graphite. Moreover, we can speculate that tube-substrate interactions might play a significantly different role in the formation of interface states when the tube lies in registry with the substrate. However, this is still an open question to be resolved in further studies.

In summary, we have related local potentials at the interfaces between MWNT's and Au, HOPG, and NiO substrates to the formation of interface states using current transients in rapidly ramped tunneling spectroscopy. Sharp variations in these potentials are observed on Au and HOPG substrates for tube diameters of around $5 \mathrm{~nm}$. These measurements indicate that the contact electronic structure is strongly influenced by variations in the electronic structure of the nanotube as a function of tube diameter. From first-principles calculations, we demonstrate that changes in $s-p$ hybridization as a function of tube diameter will lead to differences in the charge distribution at the nanotube-substrate interface. In turn, this results in modifications to the occupation of interfaces states and leads to the trends observed in the interface potentials.

The authors would like to acknowledge funding from the following agencies: DLC (NSF) and PMA (SIA/DARPA through the focus center research program established at RPI). A.R. was supported by European RTN network Contract No. HPRN-CT-2000-00128 (COMELCAN), RTD-FET program (SATUNET), and JCyL (VA28/99).
${ }^{1}$ For a review see C. Dekker, Phys. Today 52(5), 197 (1999).

${ }^{2}$ L. Chico, V.H. Crespi, L.X. Benedict, S.G. Louie, and M.L. Cohen, Phys. Rev. Lett. 76, 971 (1996).

${ }^{3}$ K. Liu, Ph. Avouris, and R. Martel, Phys. Rev. B 63, 161404 (2001).

${ }^{4}$ A. Rubio, Y. Miyamoto, X. Blase, M.L. Cohen, and S.G. Louie, Phys. Rev. B 53, 4023 (1996).

${ }^{5}$ S.J. Tans et al., Appl. Phys. Lett. 73, 274 (1998).

${ }^{6}$ Y. Xue and S. Datta, Phys. Rev. Lett. 83, 4844 (1999).

${ }^{7}$ L.C. Venema et al., Science 283, 52 (1999); A. Rubio et al., Phys. Rev. Lett. 82, 3520 (1999).

${ }^{8}$ T.W. Ebbesen and P.M. Ajayan, Nature (London) 358, 220 (1992)

9 "Short" was determined experimentally by changing filter values, etc., on the microscope and observing the effects on transients within the system.

${ }^{10}$ Individual $I-V$ spectra were smoothed using three-point cubic splines so as to remove ambiguities caused by noise at the very low currents. Electronic noise in the RHK system we use is approximately $10 \mathrm{fA}$.

${ }^{11}$ We note that the offset should be the same with and without the tube and should be equal to the work function mismatch of the tip and the substrate (i.e., the contact potential of $\mathrm{Au}-\mathrm{Pt}$, etc.).
However, to avoid artifacts due to Gibbs ringing at the digital voltage steps during ramping, the current transient is measured a little time after the step edge at which time it has significantly decreased. This results in a shift of the curve toward the $(0,0)$ intercept (steady state) by a constant related to the sample time delay. In our system this constant has been determined independently for any given sample delay. Using this sampling time constant the contact potential for the junction is recovered to within a few percent.

${ }^{12}$ See, for example, H. E. Rhoderick and R. H. Williams, MetalSemiconductor Contacts (Oxford University Press, London, 1987).

${ }^{13}$ C.-H. Kiang, M. Endo, P.M. Ajayan, G. Dresselhaus, and M.S. Dresselhaus, Phys. Rev. Lett. 81, 1869 (1998).

${ }^{14}$ We note that recent experiments show significant differences in local conductivity between nanotubes in and out of registry on HOPG. These calculations suggest that the Schottky dipole at the interface is quite small, however. See S. Paulson, A. Helser, M. Buongiorno Nardelli, R.M. Taylor II, M. Falvo, R. Superfine, and S. Washburn, Science 290, 1742 (2000).

${ }^{15}$ S. Frank, P. Poncharal, Z.L. Wang, and W.A. de Heer, Science 280, 1744 (1998); A. Bachtold et al., Nature (London) 397, 673 (1999); S. Roche, F. Triozon, A. Rubio, and D. Mayou, Phys. Lett. A 285, 94 (2001). 